\section{Benjamin Bonavida, PhD}

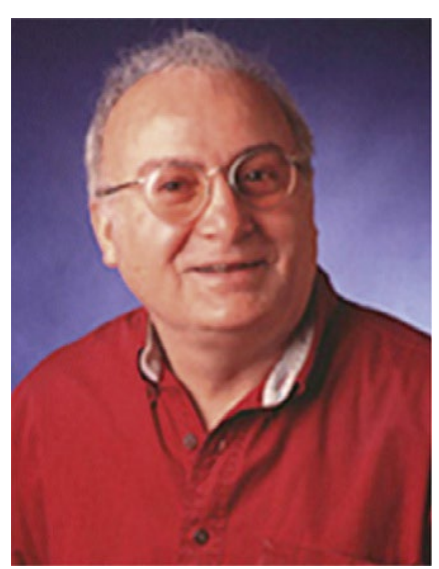

Dr. Bonavida is currently a Distinguished Research Professor at the Department of Microbiology, Immunology, and Molecular Genetics, David Geffen School of Medicine at the University of California, Los Angeles (UCLA). His research career, thus far, has focused on basic immunochemistry and cancer immunobiology. His research investigations have ranged from the mechanisms of cell-mediated killing and sensitization of resistant tumor cells to chemo-/immunotherapies, characterization of resistant factors in cancer cells, cell-signaling pathways mediated by therapeutic anticancer antibodies, and characterization of each of the gene products in the dysregulated NF- $\kappa \mathrm{B} / \mathrm{Snail} / \mathrm{YY} 1 /$ RKIP/PTEN loop in many cancers that regulate cell survival, proliferation, invasion, metastasis, and resistance. He has also investigated the role of nitric oxide in cancer and its potential anti-tumor activity. Many of these studies are centered on the clinical challenging features of cancer patients' failure to respond to both conventional and targeted therapies. He has published extensively on the research topics mentioned. He is the Series Editor for Resistance to Targeted Anti-Cancer Therapeutics published by Springer. He is also the Series Editor for the following three series: Chemotherapy-Sensitizing Agents for Cancer, Breaking Cancer Resistance to Therapeutic Antibodies, and Breaking Tolerance to AntiCancer Immunotherapy, published by Elsevier/ Academic Press.

\section{ACKNOWLEDGMENT}

The editor wishes to acknowledge the excellent assistance of Ms. Inesa Navasardyan, a student research assistant at UCLA, who has worked diligently in the editing and formatting of the various contributions of this volume and its completion for publication. 